\title{
Decreased pretreatment lymphocyte/monocyte ratio is associated with poor prognosis in stage Ib I-lla cervical cancer patients who undergo radical surgery
}

\author{
This article was published in the following Dove Press journal: \\ OncoTargets and Therapy \\ 8 June 2015 \\ Number of times this article has been viewed
}

\author{
Liang Chen ${ }^{1,2}$ \\ Fang Zhang ${ }^{3}$ \\ Xiu-gui Sheng ${ }^{2}$ \\ Shi-qian Zhang' \\ 'Department of Obstetrics and \\ Gynecology, Qilu Hospital of \\ Shandong University, Jinan, People's \\ Republic of China; ${ }^{2}$ Department of \\ Gynecological Oncology, Shandong \\ Cancer Hospital and Institute, \\ Jinan, People's Republic of China; \\ ${ }^{3}$ Department of Radiology, Provincial \\ Hospital Affiliated with Shandong \\ University, Jinan, People's Republic of \\ China
}

Background: Recently, pretreatment monocyte counts and the lymphocyte/monocyte ratio (LMR) have been proven to be significantly associated with the clinical outcomes of several types of cancer. In this study, we analyzed the prognostic significance of the LMR in stage Ib1-IIa cervical cancer patients who underwent a radical operation.

Methods: A total of 485 patients with stage Ib1-IIa cervical cancer were included in this retrospective study. We evaluated the prognostic values of the absolute lymphocyte count, absolute monocyte count, and LMR by applying receiver operating characteristic curves. Kaplan-Meier curves and multivariate Cox proportional analyses were used to determine the recurrence-free survival (RFS) and overall survival (OS).

Results: The area under the curve was 0.640 for the RFS and 0.647 for the OS using the LMR. In the univariate analysis, an elevated preoperative LMR was significantly associated with an increased RFS (hazard ratio [HR], 0.373; 95\% confidence interval [CI]: $0.247-0.563 ; P<0.001$ ), and this result remained significant in the multivariate analysis (HR, 0.439 ; 95\% CI: 0.279-0.693; $P<0.001)$. In the univariate analysis, an elevated LMR was also significantly associated with an increased OS (HR, 0.381; 95\% CI: $0.233-0.622 ; P<0.001)$, and the significance persisted in the multivariate analysis (HR, 0.417 ; 95\% CI: $0.244-0.714 ; P=0.001$ ).

Conclusion: A decreased pretreatment LMR is associated with a poor prognosis in stage Ib1-IIa cervical cancer patients who undergo a radical operation. A prospective study is warranted for further validation of our findings.

Keywords: cervical cancer, inflammation, biomarker, lymphocyte/monocyte ratio, tumor microenvironment

\section{Introduction}

Cervical cancer is the third most common cancer and the fourth leading cause of cancer-related deaths among women worldwide. ${ }^{1}$ For patients with locally advanced cervical cancer (International Federation of Gynecology and Obstetrics [FIGO] stage Ib1-IIa), radiotherapy and radical hysterectomy are effective treatment options. ${ }^{2}$ Because the classification of cervical cancer patients can help predict the risk of recurrence and thus aid in providing individualized therapies that lead to an improvement in clinical outcomes, the roles of prognostic and predictive biomarkers have become increasingly important. However, the use of biomarkers is currently limited primarily to clinicopathological prognostic factors, including the histological grade, the pathological type, the number of metastatic lymph nodes, and venous and lymphatic,
Correspondence: Shi-qian Zhang Department of Obstetrics and Gynecology, Qilu Hospital of Shandong University, Wenhuaxi Road 107, Jinan 2500 I2, People's Republic of China $\mathrm{Tel} / \mathrm{fax}+8653$ I 67626546 Email chchliang9898@163.com 
or perineural invasion levels. Only one cancer-specific antigen, the squamous cell carcinoma antigen, is used in cervical squamous carcinoma, and new biomarkers that are inexpensive and technically feasible are needed.

It is well known that inflammation plays a critical role in the pathogenesis and progression of cervical cancer. A large number of inflammatory/immune cells are found in the tumor stroma; these cells include tumor-associated macrophages (TAMs), lymphatic vascular endothelial cells, tumorinfiltrating lymphocytes (TILs ), and other inflammatory/ immune cells., ${ }^{3,4}$ These cells and the various inflammatory cytokines that they secrete interact with tumor cells and ultimately create a cancer-related inflammatory microenvironment. ${ }^{5,6}$ This environment promotes cancer angiogenesis and metastasis and alters the responses to hormones and chemotherapeutic agents. ${ }^{7}$ Furthermore, the inflammatory response to tumors may contribute to the regulation of the host immune response and lead to changes in blood constituents, specifically the neutrophilic, lymphocytic, and monocytic types of white blood cells.

The preoperative lymphocyte/monocyte ratio (LMR) has been shown to be a potential prognostic marker in patients with various types of malignancies, ${ }^{8-10}$ as have the neutrophil/ lymphocyte ratio (NLR) and the absolute count of neutrophils divided by the absolute white cell count minus the absolute count of neutrophils (dNLR). ${ }^{11,12}$ Based on the above findings, our study evaluated the effects of the preoperative LMR on recurrence-free survival (RFS) and overall survival (OS) in stage Ib1-IIa cervical cancer patients.

\section{Materials and methods}

\section{Patients and methods}

Patients with FIGO stage Ib1-IIa cervical cancer who underwent an operation between January 2004 and December 2008 at Shandong Cancer Hospital and Institute, Jinan, People's Republic of China, were included in this retrospective study. Nineteen patients (3.8\%) were excluded because they had an active infection or chronic inflammatory disease, received neoadjuvant therapy, or did not have available follow-up data; therefore, 485 patients were included in the final study. All of the patients were classified according to the FIGO clinical staging system. Clinical features, including the treatment scheme (surgery, radiotherapy, and/or chemotherapy) and histopathological information, were retrospectively obtained from the patients' records. The laboratory data, which were obtained prior to treatment and within 2 days after admission, included the absolute neutrophil count, absolute lymphocyte count (ALC), and absolute monocyte count (AMC).
This study was approved by the ethics committee of Shandong Cancer Hospital and Institute. Written informed consent was obtained from all of the patients prior to treatment.

\section{Treatment schedule}

All of the patients with FIGO stage Ib1-IIa cervical cancer underwent a radical abdominal hysterectomy and systematic bilateral pelvic lymphadenectomy. In patients with suspicious para-aortic lymph node metastasis, para-aortic lymph node dissection was performed. Postoperative platinumbased adjuvant chemotherapy with or without concurrent radiotherapy (pelvic external radiotherapy combined with vaginal intracavitary irradiation) was performed depending on the postoperative pathology. Two or three cycles of chemotherapy were permitted.

\section{Follow-up evaluation}

Follow-up was performed until June 2014. The follow-up examinations were performed at regular intervals (3-month intervals during the first 2 years, 6-month intervals in years 3-5, and annually thereafter). The examinations included a physical and pelvic examination, laboratory analyses, and imaging methods (magnetic resonance imaging, abdominal ultrasound, chest X-ray, computed tomography, and/or positron emission tomography-computed tomography).

\section{Statistical analyses}

The primary endpoint of the study was RFS (the time from diagnosis to tumor recurrence). The secondary endpoint was OS (the time from diagnosis to death by any cause). By applying a receiver operating characteristic (ROC) curve analysis, the optimal cutoff values for the ALC, AMC, and LMR were determined. The relationships between the LMR and other clinicopathological features were studied using Pearson's chi-square test or Fisher's exact test. Clinicopathological features, including the ALC, AMC, and LMR, were analyzed using Kaplan-Meier curves and compared using a log-rank test. All the clinicopathological variables associated with the RFS and OS were analyzed in the univariate analysis. The prognostic factors that had significant prognostic value in the univariate analysis were included in the multivariate Cox regression analysis. The hazard ratios (HRs) estimated from the Cox analysis were reported as relative risks with corresponding 95\% confidence intervals (CIs). The correlations between the LMR and the ALC and AMC were evaluated by applying a Pearson correlation analysis. All of the statistical analyses were performed using the Statistical Package for Social Sciences (SPSS) version 13.0 (SPSS Inc., 
Chicago, IL, USA). A two-sided $P<0.05$ was considered statistically significant.

\section{Results}

The clinicopathological variables are shown in Table 1 . The median age at the time of diagnosis was 45 years. The median follow-up time was 75 months (range, 10-118 months). The mean ALC and AMC values \pm standard error (SE) were $(1.91 \pm 0.03) \times 10^{9} / \mathrm{L}$ and $(0.52 \pm 0.01) \times 10^{9} / \mathrm{L}$, respectively. The mean LMR value \pm SE was $4.50 \pm 0.14$. Combined with the recent results from studies of various cancers, we hypothesized that the ALC, AMC, and LMR would have similar prognostic values for predicting the clinical outcome; therefore, we evaluated the effects of the ALC, AMC, and LMR by applying these values to the ROC curves. For the ALC, AMC, and LMR, the areas under the curve were 0.576, 0.611 , and 0.640 , respectively, for the RFS and 0.597, 0.603, and 0.647, respectively, for the OS. The ROC curves for the RFS are shown in Figure 1. By applying an ROC analysis, the optimal cutoff levels for the ALC, AMC, and LMR were found to be $2.16 \times 10^{9} / \mathrm{L}, 0.60 \times 10^{9} / \mathrm{L}$, and 2.87 , respectively, for RFS and $2.14 \times 10^{9} / \mathrm{L}, 0.51 \times 10^{9} / \mathrm{L}$, and 4.16 , respectively, for OS. We selected the cutoff point for RFS as the uniform point for the following survival analyses.

To investigate whether the LMR is associated with the clinical outcomes of the cervical cancer patients, univariate and multivariate analyses were performed. Figures 2 and 3 show the Kaplan-Meier curves, which reveal that an increased LMR is a consistent factor predicting less recurrence and a good prognosis in stage Ib1-IIa cervical cancer patients who undergo radical surgery $(P<0.001$, log-rank test). The tumor grades were classified as G1, G2 or G3, and the tumor stages were classified as Ib1, Ib2 or IIa in the analyses. We found significant associations between

Table I Baseline patient characteristics

\begin{tabular}{|c|c|c|c|c|}
\hline & $\begin{array}{l}\text { Cases } \\
(n=485)\end{array}$ & $\begin{array}{l}L M R \leq 2.87 \\
(n=\mid 49)\end{array}$ & $\begin{array}{l}\text { LMR }>2.87 \\
(n=336)\end{array}$ & $P$-value \\
\hline Age at diagnosis & & & & $<0.001$ \\
\hline$\leq 45$ years & $239(49.3 \%)$ & $108(72.5 \%)$ & $|3|(39.0 \%)$ & \\
\hline$>45$ years & $246(50.7 \%)$ & $4 \mathrm{I}(27.5 \%)$ & $205(61.0 \%)$ & \\
\hline Pathological type & & & & 0.362 \\
\hline SCC & $433(89.3 \%)$ & |3| (87.9\%) & $302(89.9 \%)$ & \\
\hline Non-SCC & $52(10.7 \%)$ & $18(12.1 \%)$ & $34(10.1 \%)$ & \\
\hline Tumor grade & & & & 0.165 \\
\hline GI & $50(10.3 \%)$ & $19(12.8 \%)$ & 31 (9.2\%) & \\
\hline $\mathrm{G} 2$ & $26 \mid(53.8 \%)$ & $85(57.0 \%)$ & $176(52.4 \%)$ & \\
\hline G3 & $174(35.9 \%)$ & $45(30.2 \%)$ & $129(38.4 \%)$ & \\
\hline Depth of stromal invasion & & & & 0.386 \\
\hline$<2 / 3$ & $209(43.1 \%)$ & $60(40.3 \%)$ & 149 (44.3\%) & \\
\hline$\geq 2 / 3$ & $276(56.9 \%)$ & $89(59.7 \%)$ & $187(55.7 \%)$ & \\
\hline Lymphovascular space invasion & & & & 0.098 \\
\hline Yes & $63(13.0 \%)$ & $25(16.8 \%)$ & 38 (II.3\%) & \\
\hline No & $422(87.0 \%)$ & $124(83.2 \%)$ & $298(88.7 \%)$ & \\
\hline Lymph node metastasis & & & & 0.917 \\
\hline No & $386(79.6 \%)$ & $118(79.1 \%)$ & $268(79.8 \%)$ & \\
\hline 1 & $53(10.9 \%)$ & $15(10.1 \%)$ & 38 (II.3\%) & \\
\hline 2 & II (2.3\%) & $4(2.7 \%)$ & $7(2.1 \%)$ & \\
\hline$\geq 3$ & 35 (7.2\%) & $12(8.1 \%)$ & $23(6.8 \%)$ & \\
\hline Tumor stage & & & & 0.012 \\
\hline$|b|$ & $116(23.9 \%)$ & $32(21.5 \%)$ & $84(25.0 \%)$ & \\
\hline $\mathrm{lb} 2$ & $210(43.3 \%)$ & $54(36.2 \%)$ & I56 (46.4\%) & \\
\hline Ila & $159(32.8 \%)$ & $63(42.3 \%)$ & $96(28.6 \%)$ & \\
\hline Parametrial involvement & & & & 0.022 \\
\hline Yes & $12(2.5 \%)$ & $0(0 \%)$ & $12(3.6 \%)$ & \\
\hline No & $473(975 \%)$ & 149 (100\%) & 324 (96.4\%) & \\
\hline Adjuvant therapy & & & & $<0.001$ \\
\hline None & $107(22.1 \%)$ & 47 (3I.6\%) & $60(17.9 \%)$ & \\
\hline Radiotherapy & $63(13.0 \%)$ & $6(4.0 \%)$ & $57(17.0 \%)$ & \\
\hline Chemoradiotherapy & 315 (64.9\%) & 96 (64.4\%) & $219(65.1 \%)$ & \\
\hline
\end{tabular}

Abbreviations: LMR, lymphocyte/monocyte ratio; SCC, squamous cell carcinoma. 


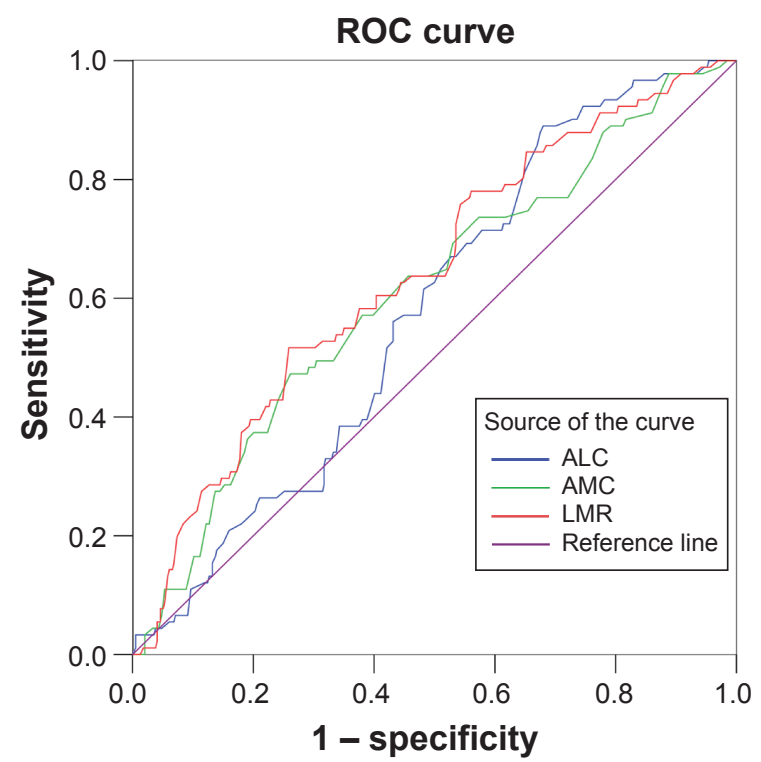

Figure I Receiver operating characteristic curves for the ALC, AMC, and LMR for RFS. Notes: ROC curves were used to maximize the prognostic value of the LMR for RFS and OS. The ROC curve is actually composed of the two-dimensional coordinates of the point group $N$ (sensitivity, I - specificity) plotted curves. Each point represents both the sensitivity and specificity of a test marker. The Youden index (ie, sensitivity + specificity -1 ), which indicates the maximum potential effectiveness of a biomarker, was chosen as the best diagnostic cutoff value in our paper. Examining RFS, the areas under the curve were $0.576,0.6 \mathrm{II}$, and 0.640 in RFS for ALC, AMC, and LMR, respectively. Diagonal segments are produced by ties.

Abbreviations: ALC, absolute lymphocyte count; AMC, absolute monocyte count; LMR, lymphocyte/monocyte ratio; RFS, recurrence-free survival; ROC, receiver operating characteristic OS, overall survival.

age, tumor grade, lymphovascular space invasion, tumor invasion depth, lymph node metastasis, tumor stage, and adjuvant therapy and the RFS and OS in the univariate analyses (all $P<0.05$ ) (Tables 2 and 3). We also observed a significant association between both the depth of stromal

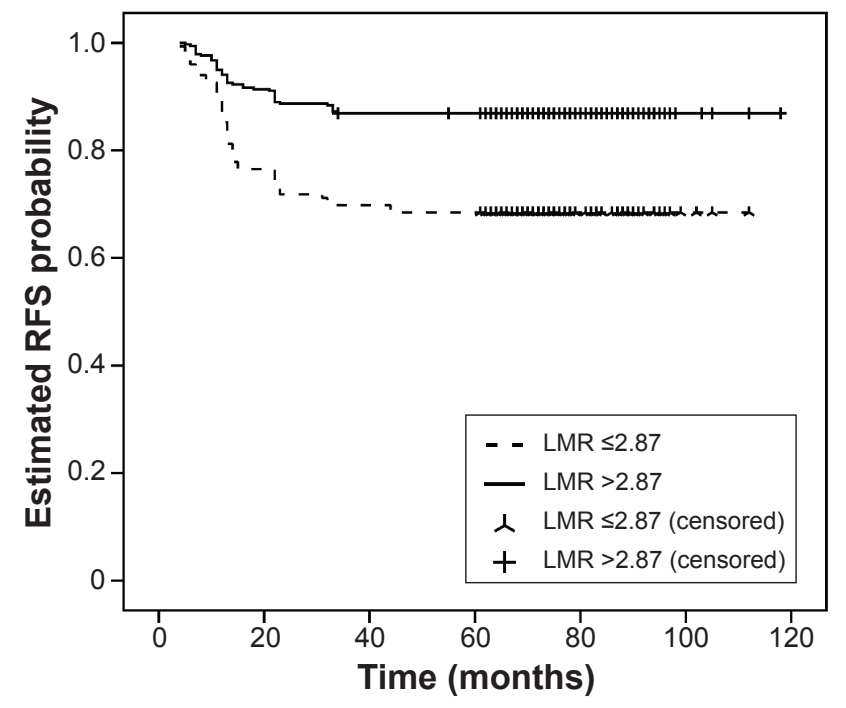

Figure 2 Kaplan-Meier curve stratified by the LMR for the RFS.

Notes: An increased LMR is a consistent factor for less recurrence in stage lb I-lla cervical cancer patients who undergo radical surgery $(P<0.00$ I, log-rank test).

Abbreviations: LMR, lymphocyte/monocyte ratio; RFS, recurrence-free survival.

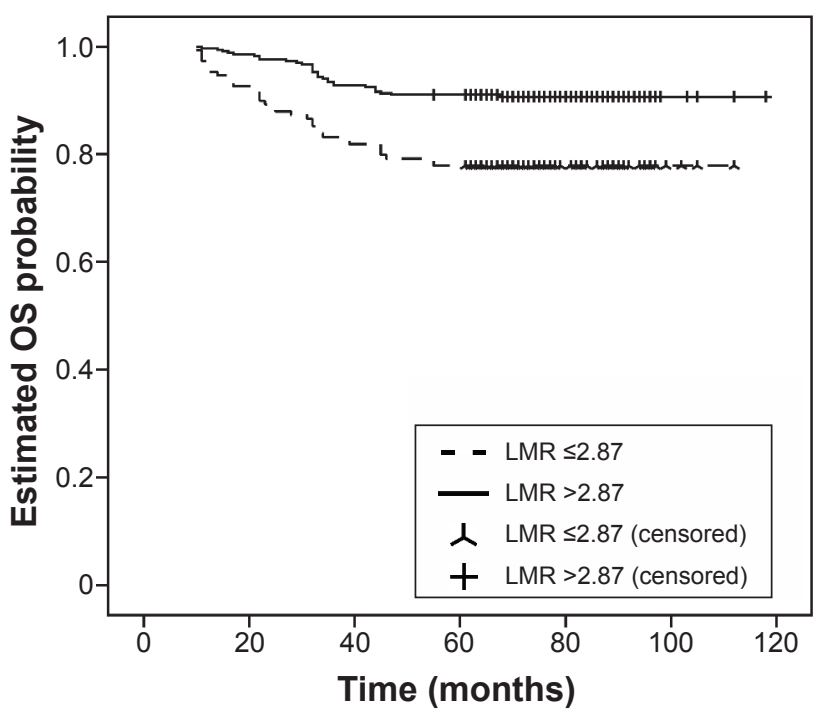

Figure 3 Kaplan-Meier curve stratified by the LMR for the OS.

Notes: An increased LMR is a consistent factor for good prognosis in stage lb I-lla cervical cancer patients who undergo radical surgery $(P<0.00 \mathrm{I}$, log-rank test).

Abbreviations: LMR, lymphocyte/monocyte ratio; OS, overall survival.

invasion and lymph node metastasis and the RFS in the multivariate analysis (all $P<0.05$ ) (Table 2). Multivariate analysis also demonstrated a similar association between lymph node metastasis and the OS $(P<0.001)$ (Table 3$)$. Other clinicopathological features were not significantly associated with the RFS or OS.

In the univariate analysis, an elevated preoperative LMR was significantly associated with an increased RFS (HR, 0.373; 95\% CI: $0.247-0.563 ; P<0.001$ ) (Table 2), and this result remained significant in the multivariate analysis (HR, 0.439; 95\% CI: $0.279-0.693 ; P<0.001$ ) (Table 2). In the univariate analysis, an elevated LMR was also significantly associated with an increased OS (HR, 0.381; 95\% CI: $0.233-0.622 ; P<0.001$ ) (Table 3), and this result remained significant in the multivariate analysis (HR, 0.417 ; 95\% CI: 0.244-0.714; $P=0.001$ ) (Table 3).

After the nonparametric tests were applied, our study showed that the LMR was significantly associated with the age at diagnosis $(P<0.001)$, tumor stage $(P=0.012)$, parametrial involvement $(P=0.022)$, and adjuvant therapy $(P<0.001)$. There were no other clinicopathological parameters associated with the LMR (all $P>0.05$ ).

Among the 485 cervical cancer patients, 91 (18.8\%) developed tumor recurrence, and 64 (13.2\%) died during the follow-up period. Tumor recurrence was diagnosed in 47 (31.5\%) out of 149 patients with an LMR $\leq 2.87$ and in $44(13.1 \%)$ out of 336 patients with an LMR $>2.87$ $(P<0.001)$. Death occurred in $33(22.1 \%)$ patients with an LMR $\leq 2.87$ and in $31(9.2 \%)$ patients with an LMR $>2.87$ $(P<0.001)$. 
Table 2 Univariate and multivariate analyses of the association of the prognostic factors and the LMR with RFS

\begin{tabular}{|c|c|c|c|c|}
\hline Factors & $\begin{array}{l}\text { Univariate analysis } \\
\text { HR }(95 \% \mathrm{Cl})\end{array}$ & $P$-value & $\begin{array}{l}\text { Multivariate analysis } \\
\text { HR }(95 \% \mathrm{Cl})\end{array}$ & $P$-value \\
\hline \multicolumn{5}{|l|}{ Age at diagnosis } \\
\hline$<45$ years & 1 & $<0.001$ & I & 0.084 \\
\hline$\geq 45$ years & $0.443(0.286-0.687)$ & & $0.657(0.408-1.058)$ & \\
\hline \multicolumn{5}{|l|}{ Pathological type } \\
\hline SCC & 1 & 0.866 & & \\
\hline Non-SCC & $0.965(0.639-1.458)$ & & & \\
\hline \multicolumn{5}{|l|}{ Tumor grade } \\
\hline GI & I & 0.024 & I & 0.276 \\
\hline G2 & $\mathrm{I} .479(1.054-2.076)$ & & $1.232(0.846-1.793)$ & \\
\hline \multicolumn{5}{|l|}{ G3 } \\
\hline \multicolumn{5}{|c|}{ Depth of stromal invasion } \\
\hline$<2 / 3$ & 1 & $<0.001$ & I & 0.043 \\
\hline$\geq 2 / 3$ & $1.574(1.264-1.961)$ & & $1.398(1.010-1.935)$ & \\
\hline \multicolumn{5}{|c|}{ Lymphovascular space invasion } \\
\hline Yes & 1 & $<0.001$ & I & 0.073 \\
\hline No & $2.718(1.704-4.335)$ & & $1.608(0.956-2.705)$ & \\
\hline \multicolumn{5}{|c|}{ Lymph node metastasis } \\
\hline No & I & $<0.001$ & I & $<0.001$ \\
\hline 1 & $1.854(1.567-2.194)$ & & $1.735(1.457-2.065)$ & \\
\hline \multicolumn{5}{|l|}{2} \\
\hline \multicolumn{5}{|l|}{$\geq 3$} \\
\hline \multicolumn{5}{|l|}{ Tumor stage } \\
\hline$|b|$ & 1 & 0.015 & I & 0.072 \\
\hline lb2 & $\mathrm{I} .425(\mathrm{I} .072-\mathrm{I} .893)$ & & I.329 (0.975-I.8I2) & \\
\hline \multicolumn{5}{|c|}{ Ila } \\
\hline \multicolumn{5}{|c|}{ Parametrial involvement } \\
\hline Yes & 1 & 0.385 & & \\
\hline No & $0.418(0.058-2.998)$ & & & \\
\hline \multicolumn{5}{|l|}{ Adjuvant therapy } \\
\hline None & I & 0.001 & I & 0.816 \\
\hline Radiotherapy & $1.778(1.280-2.468)$ & & $0.946(0.59|-| .5 \mid 4)$ & \\
\hline \multicolumn{5}{|c|}{ Chemoradiotherapy } \\
\hline \multicolumn{5}{|c|}{ LMR } \\
\hline$\leq 2.87$ & 1 & $<0.001$ & I & $<0.001$ \\
\hline$>2.87$ & $0.373(0.247-0.563)$ & & $0.439(0.279-0.693)$ & \\
\hline
\end{tabular}

Abbreviations: $\mathrm{Cl}$, confidence interval; HR, hazard ratio; LMR, lymphocyte/monocyte ratio; RFS, recurrence-free survival; SCC, squamous cell carcinoma.

For further analysis of the prognostic value of LMR, we explored the mean value (4.50) as the cut-off point for testing. An elevated preoperative LMR was significantly associated with increased RFS in the univariate (HR, $0.485 ; 95 \% \mathrm{CI}$, $0.295-0.797 ; P=0.004)$ and multivariate $(0.531 ; 95 \% \mathrm{CI}$, $0.319-0.884 ; P=0.015)$ analysis. Additionally, a significant association between LMR and OS was observed in the univariate (HR, $0.393 ; 95 \% \mathrm{CI}, 0.210-0.736 ; P=0.004)$ and multivariate (HR, $0.400 ; 95 \% \mathrm{CI}, 0.211-0.758 ; P=0.005)$ analysis. The recurrence rate was $22.8 \%(71 / 312)$ in patients with an LMR $\leq 4.50$ and $11.6 \%(20 / 173)$ in patients with an LMR $>4.50$. Death occurred in $52(16.7 \%)$ patients with an LMR $\leq 4.50$ and in 12 (6.9\%) patients with an LMR $>4.50$.

In the univariate analysis, an elevated ALC was significantly associated with an increased RFS (HR, 0.288; 95\% CI: 0.149-0.556; $P<0.001)$ and an increased OS (HR, 0.296;
95\% CI: $0.135-0.648 ; P=0.002)$, whereas an elevated AMC was significantly associated with a decreased RFS (HR, 2.253; 95\% CI: $1.493-3.401 ; P<0.001)$ and a decreased OS (HR, 2.196; 95\% CI: $1.344-3.589 ; P=0.002$ ). In the multivariate analysis, an elevated preoperative ALC was significantly associated with an increased RFS (HR, 0.403; 95\% CI: 0.199-0.815; $P=0.011)$ and an increased OS (HR, $0.407 ; 95 \%$ CI: $0.177-0.939 ; P=0.035)$, whereas an elevated AMC was significantly associated with a decreased RFS (HR, 1.750; 95\% CI: 1.127-2.715; $P=0.013$ ) and OS (HR, 1.862; 95\% CI, 1.109-3.126; $P=0.019$ ). The LMR was not included in the multivariate analysis simultaneously because this value was derived from the ratio of the AMC to the ALC and thus was significantly related to the AMC (correlation $r=0.432 ; P<0.001$ ) and the ALC (correlation $r=0.591 ; P<0.001)$. 
Table 3 Univariate and multivariate analyses of the association of prognostic factors and LMR with the time to OS

\begin{tabular}{|c|c|c|c|c|}
\hline Factors & $\begin{array}{l}\text { Univariate analysis } \\
\text { HR }(95 \% \mathrm{Cl})\end{array}$ & $P$-value & $\begin{array}{l}\text { Multivariate analysis } \\
\text { HR }(95 \% \mathrm{Cl})\end{array}$ & $P$-value \\
\hline \multicolumn{5}{|l|}{ Age at diagnosis } \\
\hline$<45$ years & I & 0.002 & 1 & 0.244 \\
\hline$\geq 45$ years & $0.447(0.265-0.753)$ & & $0.715(0.406-1.258)$ & \\
\hline \multicolumn{5}{|l|}{ Pathological type } \\
\hline SCC & I & 0.778 & & \\
\hline Non-SCC & $0.928(0.553-1.557)$ & & & \\
\hline \multicolumn{5}{|l|}{ Tumor grade } \\
\hline GI & 1 & 0.007 & 1 & 0.056 \\
\hline G2 & $1.783(1.175-2.707)$ & & I.574 (0.989-2.507) & \\
\hline \multicolumn{5}{|l|}{ G3 } \\
\hline \multicolumn{5}{|c|}{ Depth of stromal invasion } \\
\hline$<2 / 3$ & I & 0.001 & 1 & 0.063 \\
\hline$\geq 2 / 3$ & $1.570(1.208-2.042)$ & & $1.455(0.980-2.16 \mathrm{I})$ & \\
\hline \multicolumn{5}{|c|}{ Lymphovascular space invasion } \\
\hline Yes & I & 0.002 & 1 & 0.455 \\
\hline No & $2.451(1.392-4.317)$ & & I.269 (0.679-2.37I) & \\
\hline \multicolumn{5}{|c|}{ Lymph node metastasis } \\
\hline No & I & $<0.001$ & I & $<0.001$ \\
\hline 1 & $1.743(1.425-2.132)$ & & $1.596(1.292-1.972)$ & \\
\hline \multicolumn{5}{|l|}{2} \\
\hline \multicolumn{5}{|l|}{$\geq 3$} \\
\hline \multicolumn{5}{|l|}{ Tumor stage } \\
\hline$|b|$ & I & 0.031 & 1 & 0.203 \\
\hline lb2 & $1.454(1.034-2.046)$ & & I.27I (0.879-I.838) & \\
\hline \multicolumn{5}{|l|}{ Ila } \\
\hline \multicolumn{5}{|c|}{ Parametrial involvement } \\
\hline Yes & I & 0.642 & & \\
\hline No & $0.626(0.087-4.513)$ & & & \\
\hline \multicolumn{5}{|l|}{ Adjuvant therapy } \\
\hline None & 1 & 0.004 & 1 & 0.842 \\
\hline Radiotherapy & $1.776(1.199-2.631)$ & & $0.943(0.532-1.674)$ & \\
\hline \multicolumn{5}{|c|}{ Chemoradiotherapy } \\
\hline \multicolumn{5}{|c|}{ LMR } \\
\hline$\leq 2.87$ & I & $<0.001$ & 1 & 0.001 \\
\hline$>2.87$ & $0.381(0.233-0.622)$ & & $0.417(0.244-0.714)$ & \\
\hline
\end{tabular}

Abbreviations: $\mathrm{Cl}$, confidence interval; HR, hazard ratio; LMR, lymphocyte/monocyte ratio; OS, overall survival; SCC, squamous cell carcinoma.

\section{Discussion}

Several explanations for the association between the LMR and the clinical outcome of cancer patients have been postulated. Lymphocytes act as basic components of the immune system, and decreased lymphocytes in the blood and in the tumor stroma lead to a down regulation of the immune response against the tumor. ${ }^{13-16}$ Moreover, decreased proportions of tumor-infiltrating CD4 ${ }^{+} \mathrm{T}$-cells with reversed CD4/ CD8 ratios are significantly related to tumor growth and lymph node metastasis in cervical cancer. ${ }^{17}$ Therefore, lymphocytopenia has been found to be an independent prognostic factor for OS in cancers such as hepatocellular carcinoma and nasopharyngeal carcinoma. ${ }^{18,19} \mathrm{We}$ found that an elevated ALC was significantly associated with decreased RFS and OS in stage Ib1-IIa cervical cancer patients.
Recent studies suggest that, compared with the ALC, the neutrophil count is related to increased tumor aggressiveness. Considering the NLR and dNLR, recent studies have found that the dNLR has a similar but slightly lower prognostic value compared with the NLR. ${ }^{11,12}$ Absenger et al and Dalpiaz et al suggest that monocytes, which are included in the dNLR but not in the NLR, may be responsible for the difference. ${ }^{11,12}$ In various cancers, monocytes are associated with a poor clinical outcome and modulate the tumor microenvironment, notably by inducing an aggressive phenotype and producing survival/growth factors. ${ }^{10,20}$ Moreover, macrophages in the tumor stroma are differentiated from monocytes in human serum. By co-culturing with macrophages, the aggressiveness of cancer cell lines can be effectively enhanced. ${ }^{21}$ Patients with a low TAM density have a significantly better 
disease-free survival prognosis than those with a high TAM density. ${ }^{22}$ Tumor infiltration is significantly related to VEGF expression and microvessel density. ${ }^{4}$ Therefore, monocytes have been suggested to play a role similar to that of neutrophils in the immune response to tumors, in opposition to the role of lymphocytes. Recent studies suggest that the LMR is a prognostic factor similar to the NLR in terms of its ability to predict the clinical outcome of several cancers. ${ }^{9,10}$ Our studies show, for the first time, that an increased AMC is significantly associated with a poor prognosis in stage Ib1-IIa cervical cancer patients who undergo a radical operation.

To the best of our knowledge, this study is the first to evaluate the influence of the LMR on the RFS and OS in patients with cervical cancer. Our findings show that patients with an LMR > 2.87 seem to have a significantly longer RFS and OS, which is consistent with the results of other studies involving a variety of malignant tumors. ${ }^{9,10,19}$ The reason for this result may be that, similar to the NLR and dNLR, the LMR is believed to reflect a disorder of the antitumor immune system in cancer patients. The relationship between the LMR and the age at diagnosis $(P<0.001)$, tumor stage $(P=0.012)$, and parametrial involvement $(P=0.022)$ may also contribute to the prognostic value of the LMR. A low LMR may reflect a more serious immune system disorder and an advanced stage of cervical cancer.

In the univariate analysis, our study indicated that the $\mathrm{AMC}$ and ALC were significantly correlated with the clinical outcome in stage Ib1-IIa cervical cancer patients. We wondered whether the AMC and ALC had prognostic values similar to those of their ratio, the LMR. By applying ROC curves and a multivariate Cox regression analysis, we found that all three of these biomarkers have similar prognostic effects and that the LMR was slightly superior for predicting both the recurrence of and death from cervical cancer. The reason for its superiority may be that the LMR is derived from both the ALC and the AMC. An increased ALC was a good prognostic factor for recurrence and survival, whereas an increased AMC was a poor prognostic factor. Thus, the LMR may expand the prognostic value of these counts.

As with all retrospective studies, our study could not completely avoid a selection bias. We attempted to reduce the interference by excluding patients with an active infection, chronic inflammatory disease, or unavailable follow-up data. One of the strengths of our study is the short time interval between admission and blood collection, which occurred within 2 days after admission, thereby excluding any possible interference of treatment or drugs that could affect local inflammation. The relatively large sample size was also an advantage.

\section{Conclusion}

In summary, we have proven that an elevated LMR is a potential prognostic marker for patients with primary operable cervical cancer. Because this ratio can be easily obtained with a routine blood test, the LMR may be a useful and inexpensive biomarker for predicting the clinical outcome. A prospective study is warranted for further validation of our findings.

\section{Disclosure}

The authors report no conflicts of interest in this work.

\section{References}

1. Jemal A, Bray F, Center MM, Ferlay J, Ward E, Forman D. Global cancer statistics. CA: A Cancer Journal for Clinicians. 2011;61:69-90.

2. Landoni F, Sartori E, Maggino T, et al. Is there a role for postoperative treatment in patients with stage Ib2-IIb cervical cancer treated with neo-adjuvant chemotherapy and radical surgery? An Italian multicenter retrospective study. Gynecologic Oncology. 2014;132:611-617.

3. Gooden MJ, de Bock GH, Leffers N, Daemen T, Nijman HW. The prognostic influence of tumour-infiltrating lymphocytes in cancer: a systematic review with meta-analysis. British Journal of Cancer. 2011; 105:93-103.

4. Tsutsui S, Yasuda K, Suzuki K, Tahara K, Higashi H, Era S. Macrophage infiltration and its prognostic implications in breast cancer: the relationship with VEGF expression and microvessel density. Oncology Reports. 2005; 14:425-431.

5. Mantovani A, Allavena P, Sica A, Balkwill F. Cancer-related inflammation. Nature. 2008;454:436-444.

6. Franklin RA, Liao W, Sarkar A, et al. The cellular and molecular origin of tumor-associated macrophages. Science (New York, N.Y.). 2014; 344:921-925.

7. Del Prete A, Allavena P, Santoro G, Fumarulo R, Corsi MM, Mantovani A. Molecular pathways in cancer-related inflammation. Biochemia Medica. 2011;21:264-275.

8. Feng JF, Huang Y, Liu JS. Combination of neutrophil lymphocyte ratio and platelet lymphocyte ratio is a useful predictor of postoperative survival in patients with esophageal squamous cell carcinoma. Onco-Targets and Therapy. 2013;6:1605-1612.

9. Ni XJ, Zhang XL, Ou-Yang QW, et al. An elevated peripheral blood lymphocyte-to-monocyte ratio predicts favorable response and prognosis in locally advanced breast cancer following neoadjuvant chemotherapy. PloS One. 2014;9:e111886.

10. Hutterer GC, Stoeckigt C, Stojakovic T, et al. Low preoperative lymphocyte-monocyte ratio (LMR) represents a potentially poor prognostic factor in nonmetastatic clear cell renal cell carcinoma. Urologic Oncology. 2014;32:1041-1048.

11. Absenger G, Szkandera J, Pichler M, et al. A derived neutrophil to lymphocyte ratio predicts clinical outcome in stage II and III colon cancer patients. British Journal of Cancer. 2013;109:395-400.

12. Dalpiaz O, Pichler M, Mannweiler S, et al. Validation of the pretreatment derived neutrophil-lymphocyte ratio as a prognostic factor in a European cohort of patients with upper tract urothelial carcinoma. British Journal of Cancer. 2014;110:2531-2536.

13. Loi S, Sirtaine N, Piette F, et al. Prognostic and predictive value of tumor-infiltrating lymphocytes in a phase III randomized adjuvant breast cancer trial in node-positive breast cancer comparing the addition of docetaxel to doxorubicin with doxorubicin-based chemotherapy: BIG 02-98. Journal of Clinical Oncology: Official Journal of the American Society of Clinical Oncology. 2013;31:860-867.

14. de Jong RA, Leffers N, Boezen HM, et al. Presence of tumor-infiltrating lymphocytes is an independent prognostic factor in type I and II endometrial cancer. Gynecologic Oncology. 2009;114:105-110. 
15. Li Q, Liu L, Zhang Q, Liu S, Ge D, You Z. Interleukin-17 Indirectly Promotes M2 Macrophage Differentiation through Stimulation of COX-2/ PGE2 Pathway in the Cancer Cells. Cancer Research and Treatment: Official Journal of Korean Cancer Association. 2014;46:297-306.

16. Lorusso $\mathrm{G}$, Ruegg $\mathrm{C}$. The tumor microenvironment and its contribution to tumor evolution toward metastasis. Histochemistry and Cell Biology. 2008;130:1091-1103.

17. Sheu BC, Hsu SM, Ho HN, Lin RH, Torng PL, Huang SC. Reversed CD4/CD8 ratios of tumor-infiltrating lymphocytes are correlated with the progression of human cervical carcinoma. Cancer. 1999;86: $1537-1543$.

18. Li X, Han Z, Cheng Z, Yu J, Yu X, Liang P. Prognostic value of preoperative absolute lymphocyte count in recurrent hepatocellular carcinoma following thermal ablation: a retrospective analysis. OncoTargets and Therapy. 2014;7:1829-1835.
19. Li J, Jiang R, Liu WS, et al. A large cohort study reveals the association of elevated peripheral blood lymphocyte-to-monocyte ratio with favorable prognosis in nasopharyngeal carcinoma. PloS One. 2013;8: e83069.

20. Go SI, Kim RB, Song HN, et al. Prognostic significance of the lymphocyte-to-monocyte ratio in patients with small cell lung cancer. Medical Oncology. 2014;31:323.

21. Maess MB, Sendelbach S, Lorkowski S. Selection of reliable reference genes during THP-1 monocyte differentiation into macrophages. $B M C$ Molecular Biology. 2010;11:90.

22. Hu P, Shen H, Wang G, Zhang P, Liu Q, Du J. Prognostic significance of systemic inflammation-based lymphocyte-monocyte ratio in patients with lung cancer: based on a large cohort study. PloS One. 2014;9: e108062.
OncoTargets and Therapy

\section{Publish your work in this journal}

OncoTargets and Therapy is an international, peer-reviewed, open access journal focusing on the pathological basis of all cancers, potential targets for therapy and treatment protocols employed to improve the management of cancer patients. The journal also focuses on the impact of management programs and new therapeutic agents and protocols on

\section{Dovepress}

patient perspectives such as quality of life, adherence and satisfaction. The manuscript management system is completely online and includes a very quick and fair peer-review system, which is all easy to use. Visit http://www.dovepress.com/testimonials.php to read real quotes from published authors. 